Témoigner Témoigner. Entre histoire et mémoire

Getuigen Revue pluridisciplinaire de la Fondation Auschwitz

$127 \mid 2018$

Perpétuation de la violence après 1918

\title{
Des destins divergents : les artistes de cirque sous le national-socialisme
}

Uiteenlopende lotsbestemmingen: circusartiesten tijdens het nationaalsocialisme

\section{Annick Asso et Malte Gasche}

\section{(2) OpenEdition}

1 Journals

\section{Édition électronique}

URL : https://journals.openedition.org/temoigner/7828

DOI : $10.4000 /$ temoigner.7828

ISSN : 2506-6390

Traduction(s) :

Uiteenlopende lotsbestemmingen: circusartiesten tijdens het nationaalsocialisme - URL : https:// journals.openedition.org/temoigner/7836 [nl]

Éditeur :

Éditions du Centre d'études et de documentation Mémoire d'Auschwitz, Éditions Kimé

Édition imprimée

Date de publication : 1 octobre 2018

Pagination : 90-99

ISBN : 978-2-930953-076

ISSN : 2031-4183

Référence électronique

Annick Asso et Malte Gasche, «Des destins divergents : les artistes de cirque sous

le national-socialisme », Témoigner. Entre histoire et mémoire [En ligne], 127 | 2018, mis en ligne le 10 février 2022, consulté le 14 février 2022. URL : http://journals.openedition.org/temoigner/7828 ; DOI https://doi.org/10.4000/temoigner.7828 


\section{Varia}

\section{Des destins divergents : les artistes de cirque sous le national-socialisme}

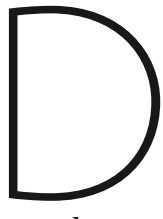

puis le XIX ${ }^{e}$ siècle, des générations de familles juives, de Sinti, de Roms et de Yéniches possédaient et exploitaient des cirques composés d'artistes venus de tous horizons, y compris des handicapés moteurs et des artistes d'ascendance africaine ou métisse. Le cirque étant une forme de divertissement très populaire, pendant la période nazie, de nombreux ont pu continuer à circuler et traverser les frontières nationales, passant par les villes et les villages.

Si les gens de cirque font, pour certains d'entre eux, figure de privilégiés parvenant à se sauver grâce à leur art, comme l'artiste germano-juive Irène Bento, de nombreux artistes juifs sont déportés et exterminés de manière tragique pendant la Shoah. En dépit de la richesse de cette complexe histoire à multiples facettes, très peu de

De ces recherches en cours émergent de nombreux témoignages de rescapés qui attestent du rôle local joué par le cirque dans les ghettos et les camps de concentration et d'extermination. La présence d'artistes de cirque permet en effet la production de spectacles malgré la pauvreté des moyens techniques. Shoah?

?

Les artistes de cirque que nous présentons ici ont tous des destins hors du commun. Ils ont laissé derrière eux des productions le plus souvent inattendues alors qu'ils étaient eux-mêmes voús à la déportation ou àlextermination : nous retrouvons les traces de perfor voús vons les traces de performances incroyables ou de numéros individuels comme
autant d'hymnes à la survie et à la résistance.

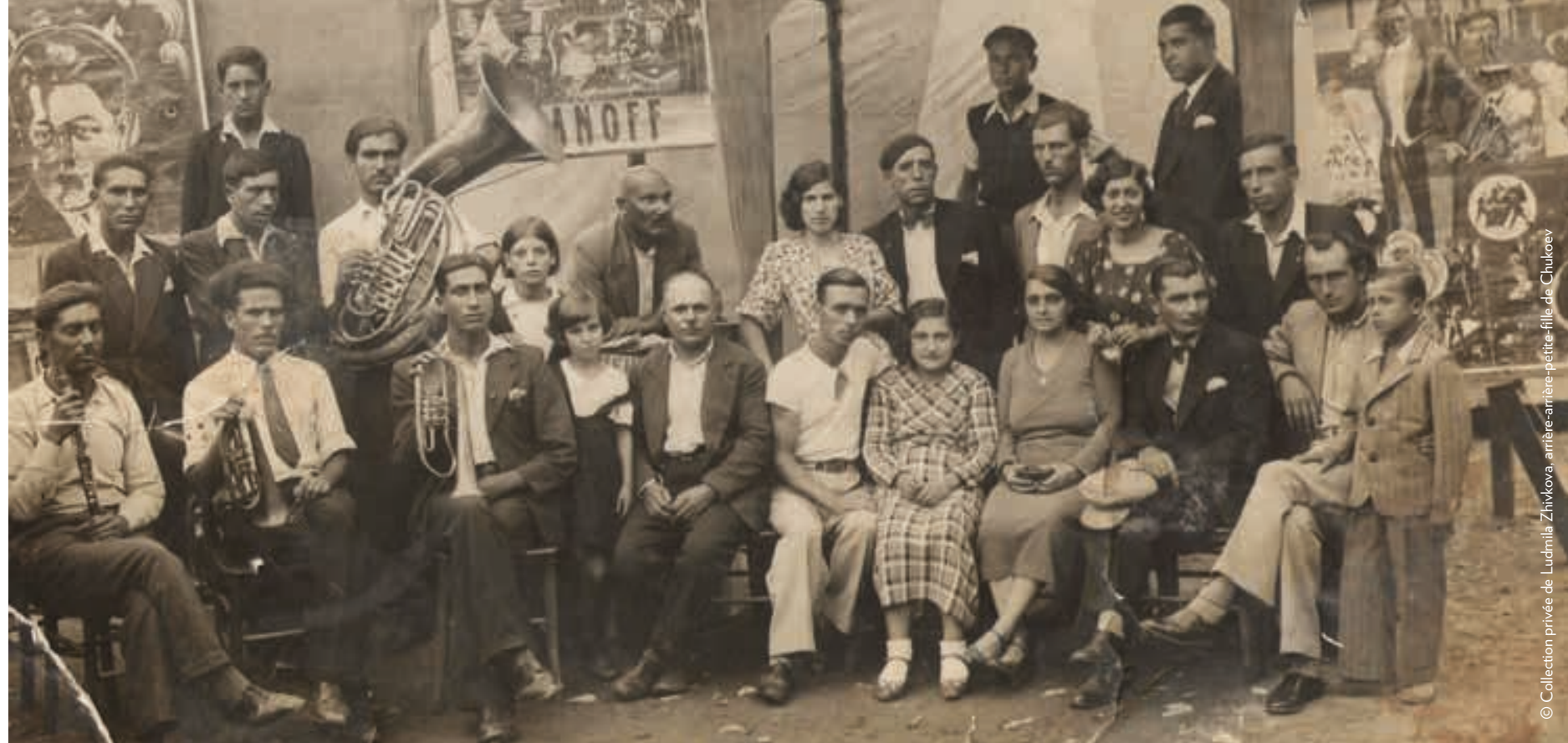

LES CLOWNS DES GHETTOS

Dans les ghettos, les spectacles de cirque sont attestés au côté des spectacles de théâtre et de cabaret. Le témoignage de Joseph Lev conservé à Yad Vashem² rapporte sa vie, enfant, au ghetto et indique que des cirques circulaient autour du rapporte sa vie, enfant, au ghetto et indique que des cirques circulaient autour du ghetto. Né en 1932 à Varsovie, toute sa famille est déportée. Il parvient à s’échapper
sous une fausse identité et travaille dans un cirque : il est chargé de s'occuper des chevaux auxquels il donne à boire, puis travaille comme assistant d'un magicien. I passe ainsi de village en village, avant de retourner en 1946 à lorphelinat, à Varsovie.

À l'intérieur des ghettos, des numéros de clown mémorables sont également relatés. Deux clowns renommés, Rubinstein, le bouffon du ghetto de Varsovie, et Hershkowitz, un chanteur de rue du Ghetto de Eódź, ont marqué les mémoires des survivants. Ils se trouvent mentionnés do de nom écrits, comme le témoisurve ianiste (Szpilman, 2003). Rubinstein est un jeune garçon espiègle qui ne craint pas de se moquer des nazis sous leur nez. Il est célèbre pour ses épigrammes. Inlassablement, il répétait: «Alle gleich!» dans une gestuelle mécanique, telle une pirouette adressée à l'ennemi et à la mort. Israël Gutman ${ }^{4}$, né à Varsovie et membre de la résistance juive du ghetto de Varsovie, décrit Rubinstein en ces termes :

Il avait l'air dérangé, comme un homme qui avait tout perdu, il portait des habits déchirés et crasseux, n’avait pas d'endroit où vivre; il ne prenait pas soin de son apparence du tout. Cétait dûàla faim quitenaillait constammentàVarsovie et finalement il s'esttransformé en clown Pouruet en clown. Pon des gens de rire. [.... Les gens n’avaient pas peur de lui. [.... Dans une société sans journaux
et sans radio, il était la figure qui représentait quelque chose de la société. Quelqưun qui
- Tucho Chukoev, Rom bulgare, musicien de cir
Second personnage, en partant de la gauche, au premier rang

(2) Témoignage vidéo $0.3 / 10450$ (3) « Tous pareils (4) Rescapé des camps de
Maidanek, Auschwitz et Mauthausen, le Pr. Pritzraël Gutman (1923-2013) a été
lun des directeurs de l'lnstitu international pour les Recherches 
ne vit pas selon les codes sociaux est appelé « anormal » et de ce point de vue, il nétait pas normal. Mais son anormalité représentait l'intelligence, et il savait comment préparer ses affirmations et ses messages codés ${ }^{5}$ (Ostrower, 2014, p. 366).

Plusieurs versions existent sur la mort de Rubinstein. L’une d'elles raconte qu'il aurait été exécuté pendant le couvre-feu : il aurait alors réussi à crier : « Je tombe avec Hitler.» Une autre version relate qu'il mourut comme il vécut: il se serait porté volontaire pour partir en déportation vers Treblinka, riant et courant tout autour volontaire pour de l'Umschlagplatz et il serait monté dans le train en continuant son numéro, tout
en riant (Engelking et Leociak, 2009, p. 594).

Yankele Hershkowitz, quant à lui, produisait des chansons satiriques qui décrivent la vie du ghetto et les injustices commises par les chefs des Judenrat et faisait figure de troubadour du ghetto (Ostrower, 2014, p. 369).

Ces numéros de clowns dans les ghettos correspondent à la tradition du bouffon juif, badkhn/badchan (Krasney, 1998) en hébreu, qui, comme l'a montré Ariela Krasney, permettait aux Juifs de développer une capacité particulière à rire des injustices pour mieux les dépasser, par le biais de l'humour.

\section{PERFORMANCES CIRCASSIENNES DANS LES CAMPS :} LE CIRQUE KONZENTRAZANI Ȧ BÖRGERMOOR

Dans les camps de travail et les camps de concentration, des performances théâtrales, musicales et plus exceptionnellement des spectacles de cirque ont lieu, le plus souvent, à la demande des nazis en guise de divertissement. Dans certains stalags et oflags, les prisonniers peuvent parfois être à l’initiative du spectacle.

En 1935, paraît, à Cologne, le témoignage du metteur en scène et acteur allemand Wolfgang Langhoff (1901-1966) Les Soldats du marais sous la schlague des nazis (Langhoff 1935). Arrêté en 1933 et torturé par la Gestapo, Langhoff part nazis (Lan jullet de lasert en juillet de la nême l'un des premiers camps de concentration alors en genèse. Langhoff y fait notamment mention du Cirque Konzentrazani, un spectacle de cirque mis en scène par lui-même, dans le camp, en août 1933. Le nom « Konzentrazani » est une allusion directe au cirque Sarrasani, créé en 1902, à Dresde, et très populaire en Allemagne. La Kommandantur avait autorisé le spectacle et le commandant et ses hommes y ont assisté personnellement : au total un public de neuf cents personnes. Langhoff développe, dans tout un chapitre, le montage du spectacle :

J'avais demandé dans chaque baraque tous ceux qui possédaient un talent quelconque, artistique, humoristique ou autre, et je fus étonné du nombre de propositions que je reçus Il y avait de tout parmi nous : des acrobates, des gymnastes, des boxeurs, des con
des chanteurs, des imitateurs de cris d’animaux, des jongleurs (Langhoff, 1935).
Lévénement suit la trame typique d'un spectacle de cirque: des sketches de cabaret, des scènes de comédie, des animaux faits de chiffons et de bouts de ficelles, des intermèdes musicaux ponctuant les scènes et préparant l'entrée du clown Auguste. Il y avait même un autre clown qui circulait parmi les spectateurs et offrait d'énormes morceaux de tourbe comme si cétait de la crème glacée. La renommée du cirque Konzentrazani est due, pour une large part, au célèbre Börgermoor Lied (Le Chant des Marais), érigé en symbole de Résistance. Les prisonniers de Börgermoor transférés vers les autres camps emportaient avec eux l'idée du cirque qui est ainsi devenu un

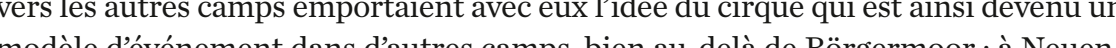
modèle dévét gamme, d’anciens soldats du Marais, Heinrich Pakullis et Ernst Saalwächter ont fait eux aussi renaître le cirque avec l'idée selon laquelle, l’on devrait toujours avoir recours
au cirque: « au cours de la vie, pendant l'agonie et dans la mort.» (Albrecht, 1984,p. 183)

\section{LE SORT DES ARTISTES DÉPORTÉS À AUSCHWITZ}

Ces performances, dans les ghettos et les camps, ont en commun de raviver le courage des déportés et de renforcer les résistances. Il ne faut pas oublier pour autant que ces spectacles ont puêtre récupérés et totalementinstrumentalisés par les nazis, tout particulièrementdans de piste, les artistes étaient torturés et/ou exterminés.

\section{Zoli Hirsch (1887-1944) et Lya Graf(1913-1941),}

\section{deux artistes de petite taille exterminés à Auschwi}

L'histoire du célèbre clown acrobate hongrois, Zoltán Hirsch, connu sous le nom de scène Zoli Hirsch, mérite dêtre rappelée à notre mémoire. Zoli Hirsch était une personne de petite taille, qui se produisait dans des spectacles de cirque et des vaudevilles. En 1942, cet artiste de renom, reconnu dans le monde entier, publie son autobiographie intitulée La grande vie d'un petit homme-artiste. Au cours de l'année 1943, les autorités hongroises font passer une série de décrets antisémites parmi lesquels certains limitent l'emploi des Juifs dans les professions artistiques. Il s'ensuit pour Hirsch une éviction du cirque hongrois Fényes. Lorsqu’en mars 1944, s'ensuit pour Hirsch une éviction du cirque hongrois Fényes. Lorsqu'en
l'armée allemande occupe la Hongrie, la situation empire rapidement l'armée allemande occupe la Hongrie, la situation empire rapidement
pour les Juifs. Hirsch tente de survivre par le colportage de livres, mais pour les Juifs. Hirsch tente de survivre par le colportage de livres, mais
il est arrêté pour cette pratique et, accessoirement aussi, sous prétexte il est arrêté pour cette pratique et, accessoirement aussi, sous pretexte qu'il porte une étoile jaune trop petite, bien que proportionnée à sa taille. Il est emprisonné et aussitôt transporté vers le camp de concentration d'Auschwitz-Birkenau. Là, Hirsch, transformé en portier et vêtu d'uniformes incroyables, eut à supporter Thumillation quotidienne de saluer les nazis qui passaient les portes du camp. Hirsch ne survit pas à Auschwitz. Il est conduit à la chambre à gaz où il périt en 1944. Dans les années 1970, cet immense artiste a été immortalisé par le Roli Zoli, un jouet mécanique représentant un clown en fer-blanc sur une moto rouge.

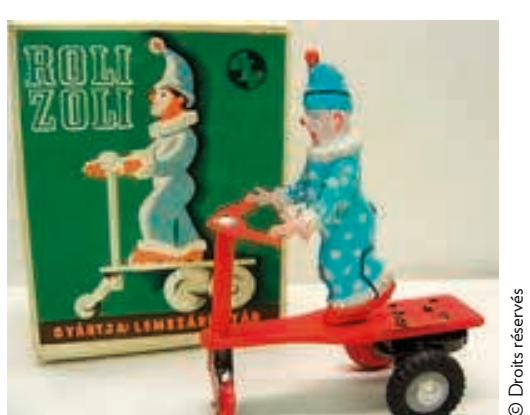




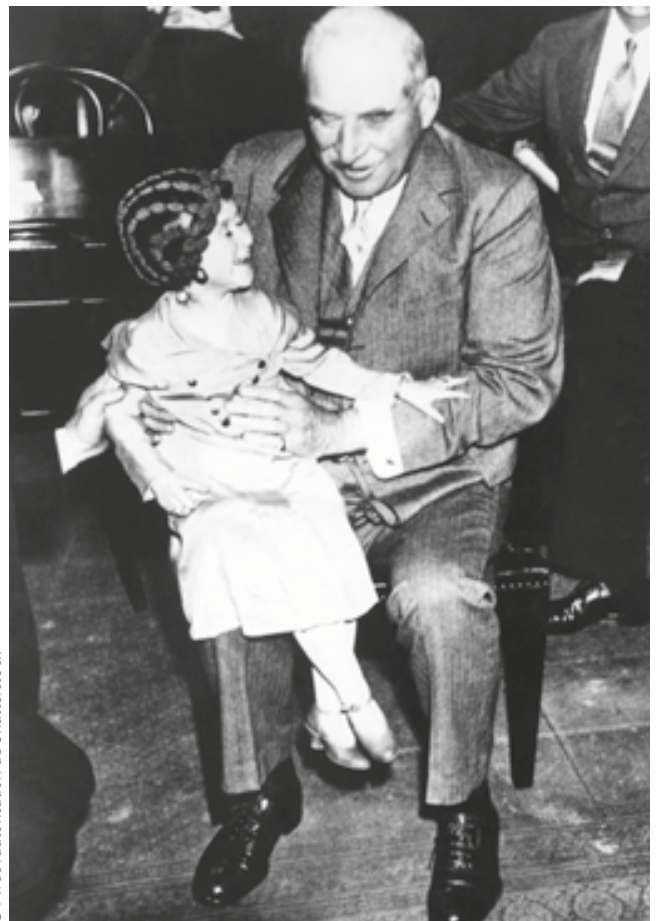

Un sort semblable est réservé à Lya Graf, de son vrai nom Lia Schwartz, une artiste de petite taille, allemande, à moitie juive d'origine, qui a travaillé dans le célèbre cirque américain Ringling and Barnum \& Bailey. Elle devient célèbre en juin 1933, lorsqu'elle monte sur les genoux du banquier de Wall Street John Pierpont Morgan Jr. dit J.P. Morgan, considéré, à l'époque, commel'homme le plus riche du monde. Laphoto de la rencontre entre «le Millionnaire et la naine a fat le tou dumonde. Grafestarrêtée pr la Gestapo en 1937 et ait le tou du mond. Grafestarrétép au camp de concentration de Sachsenhausen, près de Berlin. En 1941, elle est déportée au camp d’Auschwitz où elle trouve la mort dans les chambres à gaz.

\section{La famille Ovitz, sujets d'expérimentation} du Dr Mengele

En mai 1944, les sept frères et sœurs Ovitz, artistes de cirque juifs roumains itinérants, sont déportés à Auschwitz. Ils réchappent de justesse à la chambre àgaz par l'intervention du Docteur Josef Mengele. Celui-ci est fascinépar la possibilité de tenir à sa merci une famille entière de nains pour mener à bien ses expériences sur le nanisme et prouver que les origines juives mènent à des anomalies génétiques. Le docteur se livre avec eux à de cruelles expériences, parmi lesquelles le prélèvement de moelleépinière ou encorel'instillation d'eau bouillante dans les oreilles. Perla Ovitz (1921-2001), en proie à une forme de syndrome de Stockholm, témoigne, dans le film documentaire Liebe Perla ${ }^{6}$ qui lui a été consacré, en faveur du docteur Mengele grâce auquel elle a pu échapper à la mort dans des conditions pourtant atroces. Une réécriture du cynisme nazi envers les artistes nains est donnée par la musicienne de l'ééchiture d'Auschwitz, Fania Fénelon dans son témo ion l'orchestre d's corchestre. Le passage suivant relate l'entrée en scène d'une troupe de lilliputiens, célèbre dans toute l'Europe 7 , sous les ordres du Docteur Mengele, qui les a revêtus pour l'occasion de costumes empruntés aux déportés, des tissus magnifiques et des bijoux rutilants, qui créent une atmosphère d'autant plus terrifiante que bigarrée

Après le défilé sur la piste, ils sautent, font quelques acrobaties, en poussant des cris aigus, un numéro de clown banal, leurs mains potelées donnent des claques derisoires. C'est assez pitoyable. Nous jouons. Les SS rient, ma tâche ne m’empêche pas de regarder létrange (6) Liebe Perla, Film documentaire réalisé par Shahar
Rozen, 1999, 53 min, Germany/
Israël. spectacle. [.] Les bijoux etles soies rutilent sous le soleil, allument mille points de lumière quibougent, tourbillonnent valsent Ces créatures poussent de petits crisjoyeux essaient de chnter acc Clara Lotte ou moi. Ils ont des voix criardes, qui piaillent L'eschestre de chanter avec Corchestre chose d'irréel, de terrible. Suis-je prise de délire? Non, je vis, j’entends, je vois.
Parqué dans un coin, immobile, un public de fantômes rayés, aux yeux creux, préoccupés par sa propre mort regarde sans comprendre ce spectacle dément. Je ne sais plus ce que je joue. Je crois que plus personne ne s’en prèoccupe. Le cirque n'est plus qưun cercle où tourbillonnen des êtres difformes, où battent des mains d'enfants dont certains ont cinquante ans. Les S. rient Ces rires notre musique, cesnans tacle si eff tacle si effrayant que , fur qui couvre nos flonflons.

Schluss! Crie Mengele et tout s’arrête. Un bras retombe, un rire se transforme en grimace. La joie factice abandonne les visages qui se font craintifs. Leur maitre a-t-1 quelque chose à leur reprocher? Non, le maître a suff-

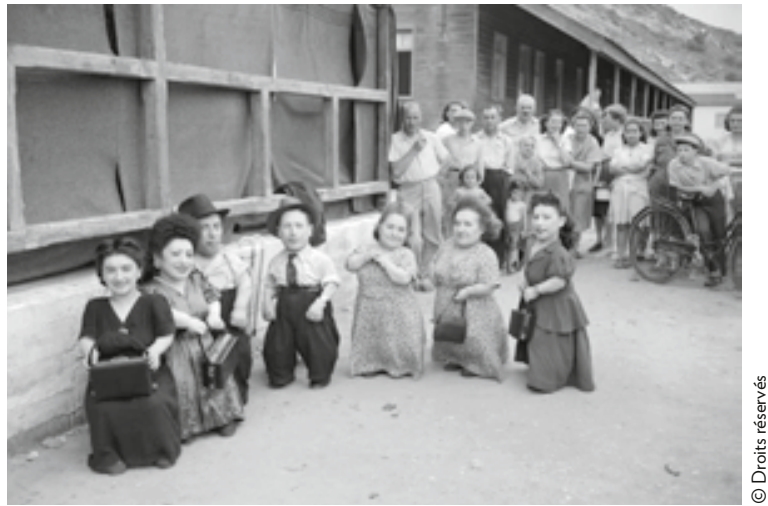

La famille Ovitz samment ri, suffisamment joui des autres. La fête est finie [...] (Fénelon, 1976, p. 319-322).

Ce sont ces destins individuels divergents qui ont aussi inspiré de nombreux écrits et fictions, comme le film $L e$ Tambour ${ }^{8}$ et apportent un éclairage particulier à l'histoire du cirque pendant la période nazie.

\section{Sioma Zubicky (1926-2014), un rescapé d'Auschwitz}

Sioma Zubicky, quant à lui, a considéré de sa responsabilité d'enseigner aux jeunes générations l'histoire de la Shoah. Pour cela, il a transcrit son expérience dans un récit autobiographique et s'est employé, jusquả la fin de sa vie, à transmettre son témoignage avec ferveur et bienveillance, en Suède, en Allemagne et dans d'autres pays européens.

Issu d'une famille de Juifs russes, Sioma Zubicky a vu le jour au sein du cirque allemand Busch. Tandis que son père Boris est un avule jour et un écuyer Zucrobate il fait preuve d'un extraordinaire talent à jouer de differents instruments de musique. L'instrument de prédilection de Zubicky est le xylophone. Après avoir joué pendant plusieurs années dans les cirques et des spectacles de variétés à travers toute l'Allemagne, la Tchécoslovaquie et la Suisse, Zubicky et sa famille déménagent à Paris, où, en juin 1940, ils sont confrontés à l'Occupation allemande. Pourtant, ils ne se cachent pas. Au contraire, Zubicky et son père font le choix d'une autre stratégie : ils se jettent pour ainsi dire dans la gueule du loup et commencent à divertir les troupes d'occupation. Du fait de leurs contacts avec la Résistance française, Zubicky et ses parents sont interrogés par la Gestapo au cours de l'automne 1943. Pendant ces interrogatoires, sa mère Annie révèle les origines juives de la famille. Son père Boris réussità śéch sper, mais Zubicky, alors âgéde 17 ans, son frère Viktor, 11 ans, Boris mère Annie sont déportés à Aus, an muméro 146.021, son frère et sa mère sont directement gazés. Au printemps 1945,
(8) Le Tambour, Film francoVolker Schalöndorff, 1979. Adapté du roman de Günter Grass, Die 
après qu'il a été libéré dans les environs de Dachau, Zubicky revient à Paris. En 1947, il accompagne Édith Piaf dans sa tournée en Suède

Ainsi, l'espace d'un court instant, le cirque confère un peu d'humanité au ghetto et au camp. Il prend une dimension sociale importante en devenant même un instrument de résistance et de survie pour certains déportés. Les formes prises par les interventions des artistes de cirque peuvent aller du simple numéro en solo pour animer le courage de leurs compatriotes, dans les ghettos ou les camps à des formes animer de productions plus vastes conme celles du cirque Konzentrazani qui integre une multitude deléting moyens du bord. Cependant, les numéros de cirque ont pu également être récupérés par le cynisme des nazis qui prennent un plaisir malsain à faire défiler leurs clowns en spectacle avant de les renfermer dans les laboratoires d'expérimentation, les torturer ou les faire disparaître dans les chambres à gaz. De ce point de vue, le cirque, pendant la période nazie, devient aussi un révélateur du sort terrible infligé aux déportés, justement par le décalage immense qu'il contribue à créer en révélant davantage l'horreur sous le masque de l'artifice.

BIBLIOGRAPHIE

- Betty M. Adelson, The Lives of Dwarfs. Their Journey from Public
Curiosity toward Social Liberation, New Brunswick, Rutgers University

- Richard Albrecht, « Zirkus Konzentrazani in: eine Modellanalyse,
Österreichische Zeitschrift für Sozziologie 9, 1 (2), 1984, p.183-190.

- Ron Chernow, The House of Morgan: An American Banking Dynasty and
the Rise of Modern Finance, New York, Grove Press, 1990 .

B

- Barbara Engelking and
University Press, 2009.

- Fania Fénelon, Sursis pour Porchestre, Paris, Stock, 1976.

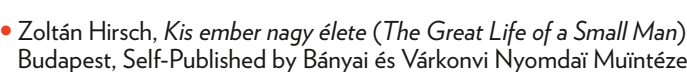
1942 .

- Anna Kérchy, «rom Showbiz To The Concentration Camp: The
Fabulous, Freakish.

- Life Of Hungarian Jewish "Dwarf "Performers, Zoli Hirsch And The
Ovitz Family, in Anna Kérchy and Andrea Zittlau Exxloring the

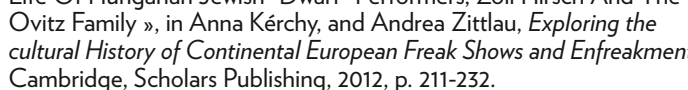

Yehuda Koren, and Eliat Negev, In Our Hearts We Were Giants. The
remarkable Story of the Lilliput Troupe - $A$ D warf Family's Survival of the remarkable Story of the Lilliput Troupe - $A$ D D
Holocaust, New York, Caroll\&\& Graf, 2004.

- Ariela Krasney, The Bachdan, Ramat Gan, Bar-llan University, 1998.

Jewgeni Kusnezow, Der Zirkus der Welt, Berlin, Henschel, 1970.

- Wolfgang Langhoff, Les Soldats du marais sous la schlague des nazis.
Treize mois de captivité dans les camps de concentration, traduit de

Treize mois de captivité dans les camps de concents
rallemand par Armand Pierhal, Paris, Plon, 1935.

- Chaya Ostrower, It kept us alive, Jerusalem, Yad Vashem, Internationa
Institute for Holocaust research, 2014.

Tien Zikksreiterin, Munich, Malik, 1997

-Wladyslaw Szpilman, Le Pianiste, Paris, Pocket, 2003.

- Sioma Zubicky, Spiel, Zirkuskind, spiel. Erimnerungen eines europäischen
Wunderkindes, Leipzig, Altberliner, 2005.
SITUATION DES CIRQUES EN ALLEMAGNE ET

EN FRANCE PENDANT LA PÉRIODE NAZIE

Une série de décisions affectent le monde du cirque pendant la période nazie.

Dès le mois de mai 1933, l'emploi de tout étranger est soumis à l'obtention d'un permis de travail officiel Le renforcementde cettelégislation après 1934 entraîne une réduction significative du nombre d'employés de tous les grands cirques allemands. Le 14 février 1935, une nouvelle dispositio impose que tous les directeurs de cirques allemands apportent la preuve de leur origine « aryenne ».

À partir de 1935-1936, le cirque n’échappe pas à l'aryanisation des arts qui se manifeste par une restriction des noms de scène à consonance étrangère, l'interdiction concernant le port de costumes, la présence sur scène d'hommes noirs, de personnes d'origines mêlées ou encore la participation de Juifs dans des spectacles « culturels allemands ». Les numéros avec des handicapés physiques sont également interdits bien que les personnes $\mathrm{d}$ petites ou grandes tailles soient toujours autorisées. Présentés comme des « nains » et des « geeants », ils incarnent des personnages de mythes et légendes germaniques.

Parallèlement les grands cirques allemands, comme Sarrasani, Krone ou Hagenbeck, bénéficient de taxes allégées et d'autorisations de tournées.

Les cirques augmentent de manière très significative leur production en Allemagne nazie au cours de la Seconde Guerre mondiale. En 1941, on comptabilise un total de 30 millions de spectateurs de cirque pour les vingt cirque les plus fréquentés d'Allemagne. En effet, les divertissements de cirque son un dérivatif utilisé pour apaiser la population en cette période de guerre. De plus, les shows patriotiques participent pleinement de la propagande.

Les mesures administratives prises à l'encontre des gens du cirque a cours des années trente ne sont pas le seul apanage de l'Allemagne nazie. Il en est de même en France, où les petits cirques familiaux constituent une tradition de longue date et font partie intégrante de la vie culturelle en milieu rural. Pourtant, le gouvernement français prend la décision de restreindre la libre circulation des populations dites nomades et, avan même que la France ne soit occupée par les troupes de la Wehrmacht, un décret du ministre de l'Intérieur ordonne l'internement de tous les groupes nomades au camp de Rivesaltes.
BIBLIOGRAPHIE

- Raymond Gurême et lsabelle Ligner, Interdit aux nomades,
Paris, Calmann-Lévy, 2011:

- Anna Lipphardt, «Spielraum
des Globalen: Deutschland und der Zirkus, , in llfiend Vermessung der Globalisierung. Pulturwissenschafftiche Winter GmbH Heidelberg. 2008, p. 168-171.

- Hans Scheugl, Show Freaks \&
Monster, Köln, Die Sammlung

Monster, Köln, Die Sa
Felix Adonos, 1975. 


\section{Varia}

IRĖNE BENTO, SAUVÉE GRÂCE AU CIRQUE ALTHOFF

Irène Bento est une artiste germano-juive, qui a survécu à la Shoah en se cachant dans le cirque allemand Althoff de 1943 à 1945. A la demande de son époux, Peter Bento, clown employé par le cirque Althoff, le jeune directeur Adolf Althoff accepte de dissimuler Irène, son fils Peter Jr., sa sœur Gerda et sa mère Alice. Irène rejoint ainsi le trio des clowns Bento et devient l'assistante de Maria Althoff, l'epouse d'Adolf, dins le dressager la Gestapo, la famille passe d'un compartiment à l'autre du wagon de cirque. Depuis 1995, 'l'État d'Israël a décerné le titre de Justes parmi les Nations à Adolf
et Maria Althoff pour avoir sauvé la famille Bento.

La vie d'Irène Bento, Irène Danner de son nom de jeune fille a été mise en scène par le collectif allemand Projektgruppe im Nationalsozialismus sous la forme d'une lecture-performance au Musée d'Etat de Berlin- Musée des Cultures européennes, lors de la Journée internationale des musées, le 21 mai 2017. Au

cours de cette représentation, des extraits du livre d'Ingeborg Prior, Der Clown und die Zirkusreiterin (Le Clown et lécuyère) ont été lus et interprétés visuellement à travers le jonglage, la pantomime et le trapèze.

Le succès de laperformance du groupe Projektgruppe im Nationalsozialismus est une belle preuve de la capacité des arts du spectacle à défier durablement l'effacement des mémoires au fil du temps qui passe et emporte les témoins directs de la Shoah. Des témoignages tels que celui d'Irène Bento portés à la 21 mai 2017 scène comportent à ce titre une haute dimension éducative.

www.divergingfates.eu, site Web interactif, présente des récits de vie, des images et des documents choisis, des listes d'archives, une des diocres une bibliographie et un sondage pour ce programme de recherche interdisciplinaire et international débuté en 2017, sous la direction du Dr Malte Gasche, qui se poursuit sur une durée de deux ans, jusqu'en 2019. En documentant la vie, la mort, mais aussi les stratégies de survie des gens du cirque en Europe souslejougnazi,l'objectifdece programme de recherche est de rendre hommage à ces artistes oubliés de l'histoire de la Shoah pour mieux porter cettehistoire encorepeuétudiée àlaconnaissance du public scolaire et du grand public.
Le projet Diverging Fates s'est poursuivi par a tenue d'un second colloque de portée internationale sur la thématique a Forgotten Cosmopo-

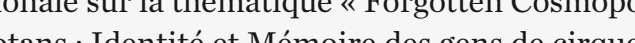
tin : Identité et Mémoire des gens de cirques tinérants en Europe » à Montpellier les 5 et avil 2018 sous la directiondes Dr Malte Gasche Pr Philippe Goudard, Dr Annick Asso et du Dr Alix de Morant.

En partenariat avec l'Alliance internationale pour la mémoire de l'Holocauste, la Fondation pour la Mémoire de la Shoah, la Fondation Kone (Finlande) et les Centres de recherche Centre for Nordic Studies, Helsinki et RIRRA 21 de l'Université Paul Valéry-Montpellier 3.

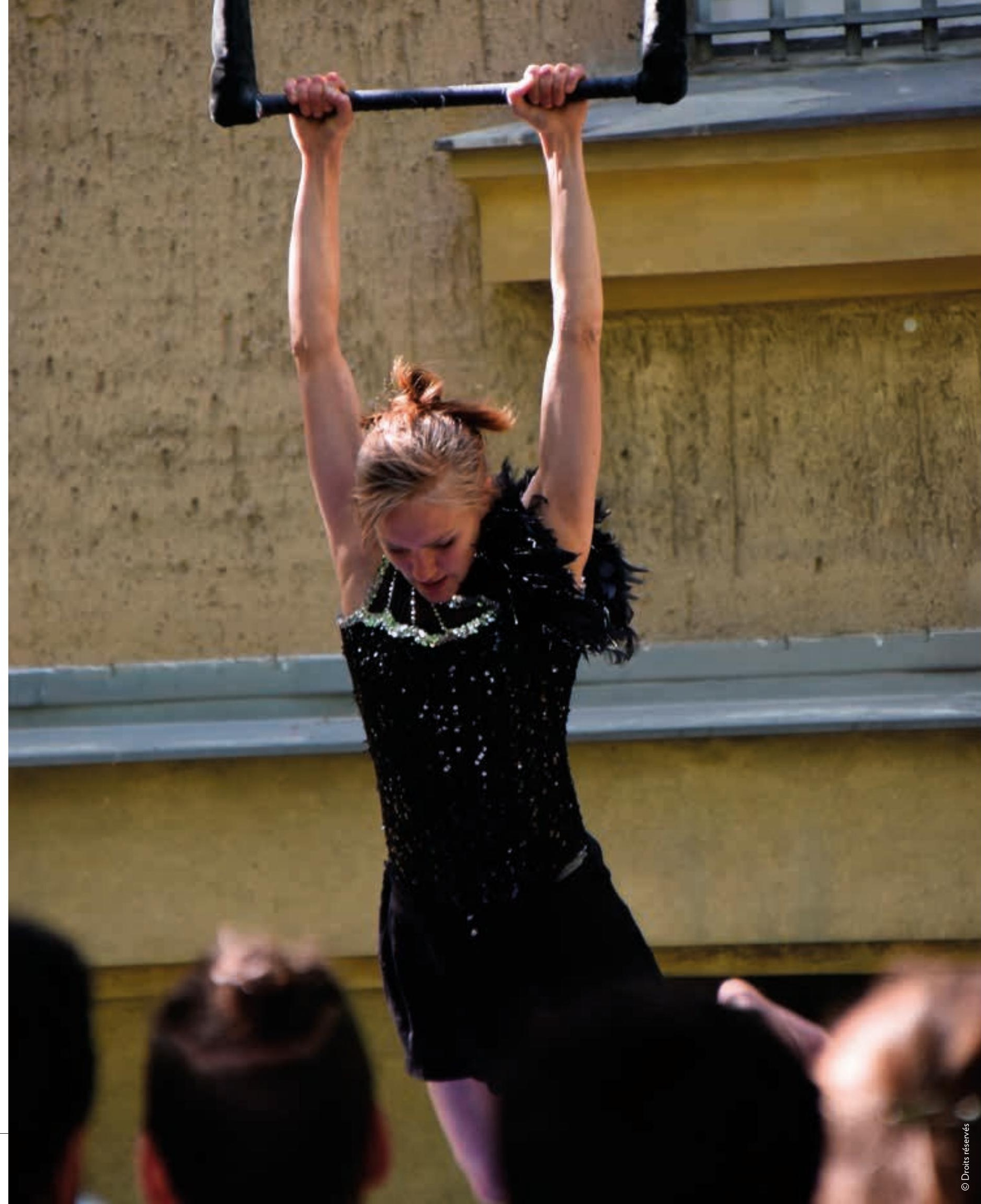

